COPYRIGHT @ 2019 INTERNATIONAL JOURNAL OF SCIENCE DENTISTRY | AVAILABLE ONLINE

http://www.periodicos.uff.br/index

\title{
TRATAMENTO CIRÚRGICO DE MALFORMAÇÃO ARTERIOVENOSA DA MUCOSA JUGAL: RELATO DE CASO
}

\section{SURGICAL TREATMENT OF ARTERIOVENOUS MALFORMATION OF BUCCAL MUCOSA: CASE REPORT}

\section{Fernanda Britto de Melo Silva}

Residente de Cirurgia Oral e Maxilofacial do Hospital Federal dos Servidores do Estado do Rio de Janeiro - HFSE e da Universidade Federal Fluminense - UFF.

\section{Rafael Mérola Corrêa}

Residente de Cirurgia Oral e Maxilofacial do Hospital Federal dos Servidores do Estado do Rio de Janeiro - HFSE e da Universidade Federal Fluminense - UFF.

\section{Flávio Merly}

Staff do Serviço de Cirurgia e Traumatologia Buco Maxilo Faciais do Hospital Federal dos Servidores do Estado do Rio de Janeiro - HFSE e Professor da disciplina de Diagnóstico Bucal da Universidade Federal Fluminense - UFF.

\section{Suelen Cristina Sartoretto}

Professora das disciplinas de Cirurgia Bucal, Semiologia e Anatomia da Faculdade de Odontologia da Universidade Iguaçu - UNIG.

Instituição na qual o trabalho foi realizado: Hospital Federal dos Servidores do Estado do Rio de Janeiro/RJ

Categoria: Relato de caso

Informações do autor principal:

Fernanda Britto de Melo Silva

Rua Ministro Viveiros de Castro, 82

Copacabana- Rio de Janeiro- RJ, Brasil.

CEP: 22021010

Email: fernandabrittodemelo2@hotmail.com

Telefone: +5521998177665 
COPYRIGHT @ 2019 INTERNATIONAL JOURNAL OF SCIENCE DENTISTRY | AVAILABLE ONLINE http://www.periodicos.uff.br/index

\title{
Resumo
}

As malformações arteriovenosas (MAV) são alterações congênitas dos vasos sanguíneos e, em casos raros, surgem apenas na idade adulta. A MAV apresenta comunicação direta entre uma artéria e uma veia que passam pelo leito capilar. Elas podem ocorrer em qualquer área do corpo e têm início e progressão graduais. O objetivo deste estudo é apresentar um caso cirúrgico de malformação arteriovenosa envolvendo a mucosa jugal direita de um paciente masculino de 16 anos de idade, tratado cirurgicamente em conjunto com o serviço de Cirurgia Vascular. O resultado pósoperatório foi satisfatório e a inter-relação entre os serviços de Cirurgia Maxilofacial e Vascular foi essencial para a realização de um procedimento cirúrgico mais seguro e previsível.

Palavras-chave: Malformação arteriovenosa; cirurgia Maxilofacial; interdisciplinaridade

\begin{abstract}
The arteriovenous malformations (AVM) are congenital alterations of the blood vessels, and in rare cases, they develop only in adulthood. AVM present direct communications between an artery and a vein bypassing the capillary bed. They can occur at any area of the body and have gradual onset and progression. The aim of this study is to present a surgical case of arteriovenous malformation involving the right buccal mucosa of a 16year-old male, which was managed with Vascular Surgery service. The postoperative result was satisfactory and interrelationship between Maxillofacial and Vascular Surgery services was essential for a safer and more predictable surgical procedure.
\end{abstract}

Key-words: Arteriovenous malformation; Maxillofacial Surgery; interdisciplinarity 


\section{Introdução}

As malformações arteriovenosas (MAVs) são patologias congênitas caracterizadas pela presença de vasos anormais (artérias, veias e capilares) formando shunts arteriovenosos, com retorno venoso precoce e arterializado (LEE, B.B., 2013). Elas são derivadas de erros na morfogênese vascular entre a quarta e sexta semanas de gestação e são completamente distintas dos hemangiomas, tumores benignos com ciclo de vida proliferativo e de involução bem definidos (KOHOUT, M.P., 1998).

Com base nas características endoteliais, Mulliken e Glowacki (1982) classificaram as lesões vasculares em hemangioma - tumor vascular - e malformações vasculares. Ambas as categorias de lesões vasculares apresentam etiologias e características clínicas diferentes.

Hemangiomas são tumores vasculares que demonstram hiperplasia endotelial que aumenta por rápida proliferação celular. Estes normalmente estão ausentes no nascimento, mas proliferam durante o primeiro ano de vida e depois involuem. Em contrapartida, as malformações vasculares são estruturas congênitas com taxa normal de renovação das células endoteliais, presentes no nascimento, mas geralmente se tornam visíveis posteriormente (LEE, B.B., 2013).

O rápido aumento das malformações geralmente é desencadeado por trauma ou alterações hormonais durante a puberdade ou gravidez. O aumento dessas lesões ocorre devido à alteração na pressão e fluxo, dilatação dos canais vasculares, desvio e proliferação colateral. Nesse sentindo, devido às características do fluxo sanguíneo, as malformações vasculares podem ser divididas em lesões de baixo fluxo e alto fluxo. As lesões de baixo fluxo incluem malformações capilares, linfáticas e venosas, enquanto as de alto fluxo incluem lesões arteriais e MAV (KUMAR, A., 2017).

As MAVs são lesões raras, representando apenas 1,5\% dentre as anormalidades vasculares e apresentam 50\% de ocorrência na região bucomaxilofacial, apresentando início e progressão graduais (SU, L.X., 2014). A MAV é considerada a forma mais desafiadora e/ou com risco de vida dentre as patologias vasculares.

REVISTA FLUMINENSE DE ODONTOLOGIA - ANO XV - No 51 - Janeiro / Junho 2019 
COPYRIGHT @ 2019 INTERNATIONAL JOURNAL OF SCIENCE DENTISTRY | AVAILABLE ONLINE http://www.periodicos.uff.br/index

O diagnóstico de imagem das lesões vasculares pode ser realizado através dos seguintes exames: ultrassom com Doppler colorido, tomografia computadorizada (TC), ressonância magnética e angiografia por ressonância magnética. Nesse sentindo, o tipo de malformação vascular pode ser identificado, bem como os vasos de drenagem e alimentação dilatados. A ressonância magnética pode ser usada como uma excelente técnica no diagnóstico, pois mostra uma boa representação da estrutura vascular, permitindo a diferenciação entre lesões de alto e baixo fluxo (LEE, B.B., 2013).

A embolização transarterial ou a ligadura das artérias alimentadoras são abordagens incorretas e podem resultar na progressão da lesão. Além disso, esses procedimentos impediriam o acesso endovascular futuro às lesões pela via arterial e, portanto não são indicados (SU, L.X., 2014). Assim, para as lesões cirurgicamente acessíveis, a ressecção cirúrgica pode ser a opção de tratamento escolhida, com controle pós operatório satisfatório. A escleroterapia ou embolização pré-operatória pode complementar a excisão cirúrgica subsequente, reduzindo a morbidade e definindo as bordas da lesão. Essa abordagem combinada é considerada uma excelente escolha para um resultado curativo (LEE, B.B., 2013).

O objetivo deste estudo é apresentar um caso cirúrgico de malformação arteriovenosa envolvendo a mucosa jugal direita de um paciente masculino de 16 anos de idade, tratado cirurgicamente em conjunto com o serviço de cirurgia vascular.

\section{Considerações éticas}

Os procedimentos empregados neste trabalho seguiram os padrões éticos propostos pela Declaração de Helsinque de 1975, revisada em 2000. O paciente foi tratado no Hospital Federal dos Servidores do Estado do Rio de Janeiro conveniado com a Universidade Federal Fluminense através da Residência em Cirurgia Oral e Maxilofacial com os termos, de consentimento livre e esclarecido e de uso de imagens e dados para publicação científica, assinados.

O estudo foi escrito seguindo as Diretrizes do Relatório de Caso Clínico (CARE, www.care-statement.org).

\section{Relato de caso}

REVISTA FLUMINENSE DE ODONTOLOGIA - ANO XV - No 51 - Janeiro / Junho 2019 
COPYRIGHT @ 2019 INTERNATIONAL JOURNAL OF SCIENCE DENTISTRY | AVAILABLE ONLINE http://www.periodicos.uff.br/index

Paciente R.M.S, 16 anos de idade, sexo masculino, procurou o Serviço de Cirurgia Oral e Maxilofacial do Hospital Federal dos Servidores do Estado do Rio de Janeiro com queixa principal de aumento de volume em região facial do lado direito com tempo de evolução de 08 anos.

Ao exame clínico apresentava assimetria facial com aumento de volume na mucosa jugal direita, estendendo-se com edema no lado ipsilateral da face (Figura 1A e 1B). Nenhum histórico médico significativo foi relatado pelo paciente e pelos pais. Não houve envolvimento dentário ou qualquer alteração na tonalidade da pele.

A Angiografia por ressonância magnética cervical e facial foi realizada em Centro Cirúrgico pela equipe de Cirurgia Vascular e confirmou o conteúdo predominantemente venoso da lesão, sem importante componente arterial envolvido, apresentando algumas estruturas venosas originadas da lesão facial no lado direito e convergindo para a veia jugular externa (Figura 1C). As imagens obtidas na Ressonância magnética (Figura 1D) justificavam o aumento de volume observado clinicamente e o paciente então foi liberado para abordagem cirúrgica pela equipe de Cirurgia Maxilofacial.

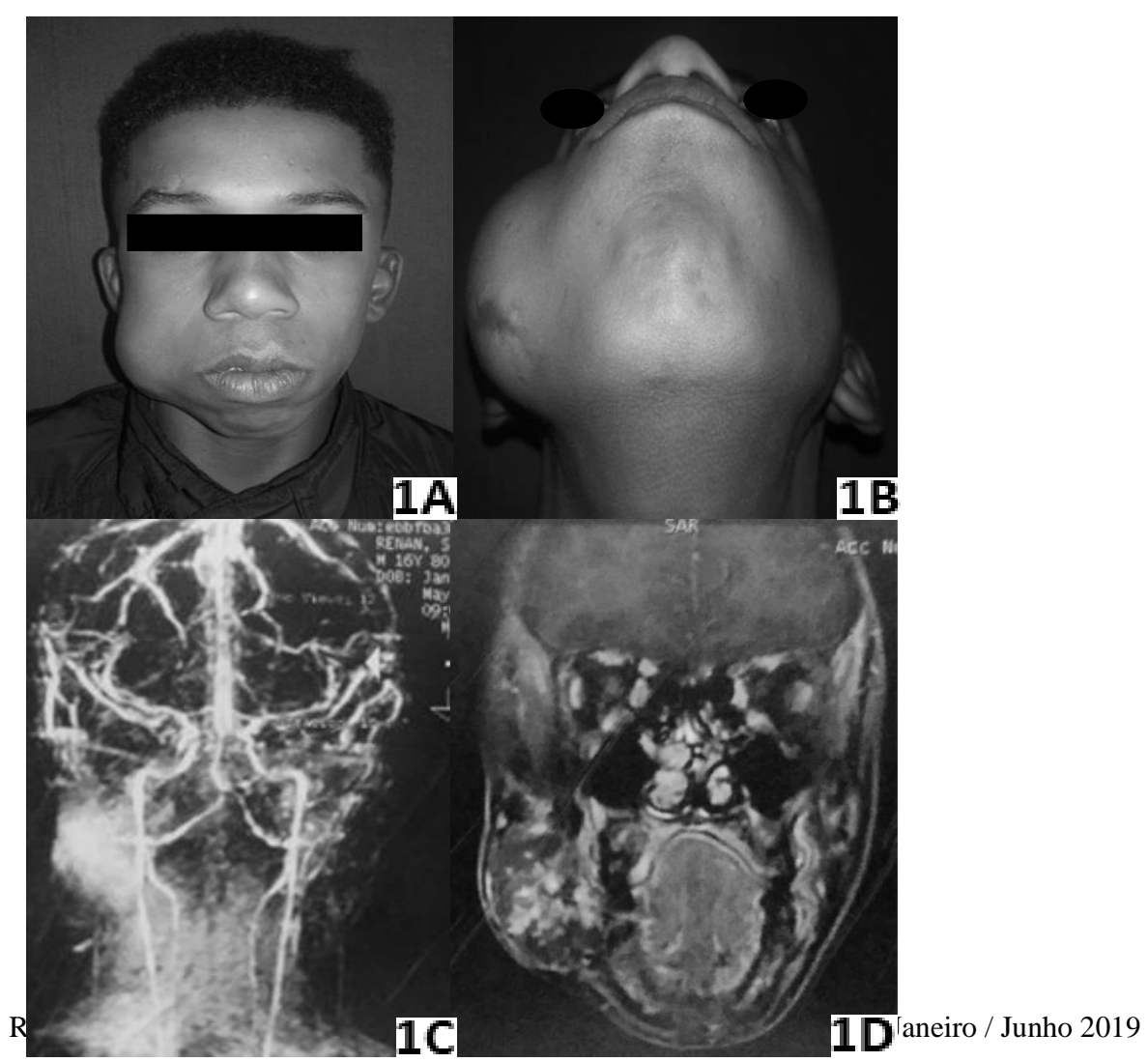


COPYRIGHT @ 2019 INTERNATIONAL JOURNAL OF SCIENCE DENTISTRY | AVAILABLE ONLINE http://www.periodicos.uff.br/index

Figura 1. A) Vista frontal evidenciando aumento de volume na mucosa bucal direita, estendendo-se com edema no lado ipsilateral da face; B) Vista inferior demonstrando aumento de volume e assimetria facial importante e importante no lado direito; C) Angiografia por ressonância magnética cervical e facial, apresentando estruturas venosas originadas da lesão facial no lado direito convergindo para a veia jugular externa; D) Ressonância magnética da face justificando o aumento de volume observado clinicamente.

O paciente foi submetido à terapia esclerosante local sem resultados satisfatórios, após, o planejamento cirúrgico estabelecido foi de biopsia excisional da lesão.

O procedimento cirúrgico foi realizado sob anestesia geral e intubação nasotraqueal. Foi realizada marcação das linhas de incisão (Figura 2A) e infiltração com lidocaína 2\% e epinefrina 1:100.000 para vasoconstricção local. O acesso submandibular estendido do lado direito foi realizado com profundidade até a camada subcutânea (Figura 2B) e através de dissecção e ligadura de pequenos vasos, o componente de malformação venosa foi ressecado (Figura 2C), sem intercorrências cirúrgicas e com bom controle hemostático. Como tratamento coadjuvante um hemostático à base de carboximetilcelulose aplicado a fim de auxiliar no controle do sangramento pós-operatório (Figura 2D).

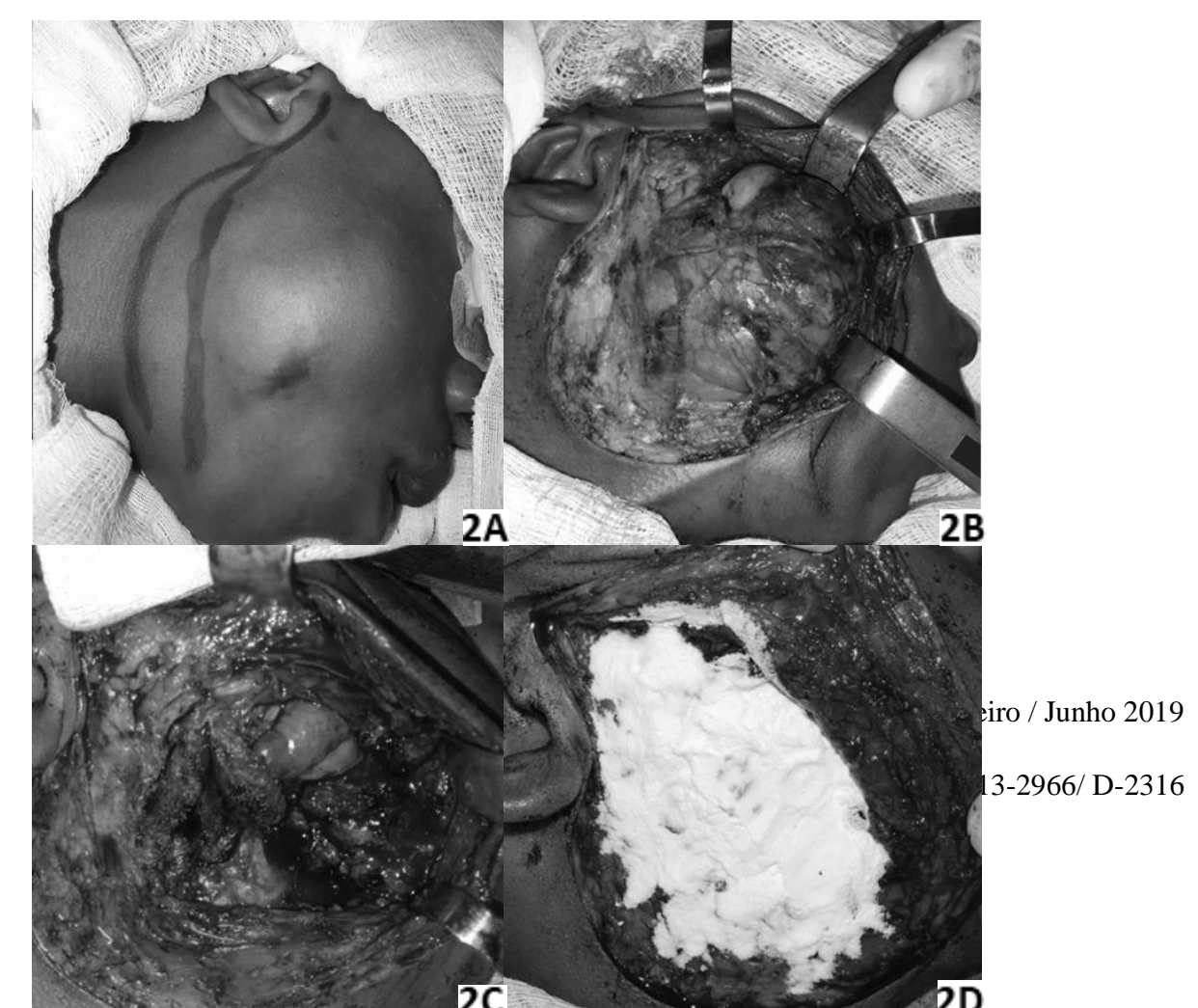


COPYRIGHT @ 2019 INTERNATIONAL JOURNAL OF SCIENCE DENTISTRY | AVAILABLE ONLINE http://www.periodicos.uff.br/index

Figura 2. A) Visão transoperatória mostrando a demarcação de acesso previamente à incisão; B) Aspecto da MAV por acesso submandibular estendido a nível de camada subcutânea; C) Vista após excisão da lesão; D) Aplicação de hemostático à base de carboximetilcelulose para evitar sangramento no pósoperatório.

Durante o acompanhamento pós-operatório imediato, o paciente apresentou boa evolução, sem complicações na ferida cirúrgica e com resolução das queixas prévias. Em acompanhamento de um ano, ao exame clínico e de imagem o paciente segue sem alterações ou sintomatologia (Figura 3A e 3B).

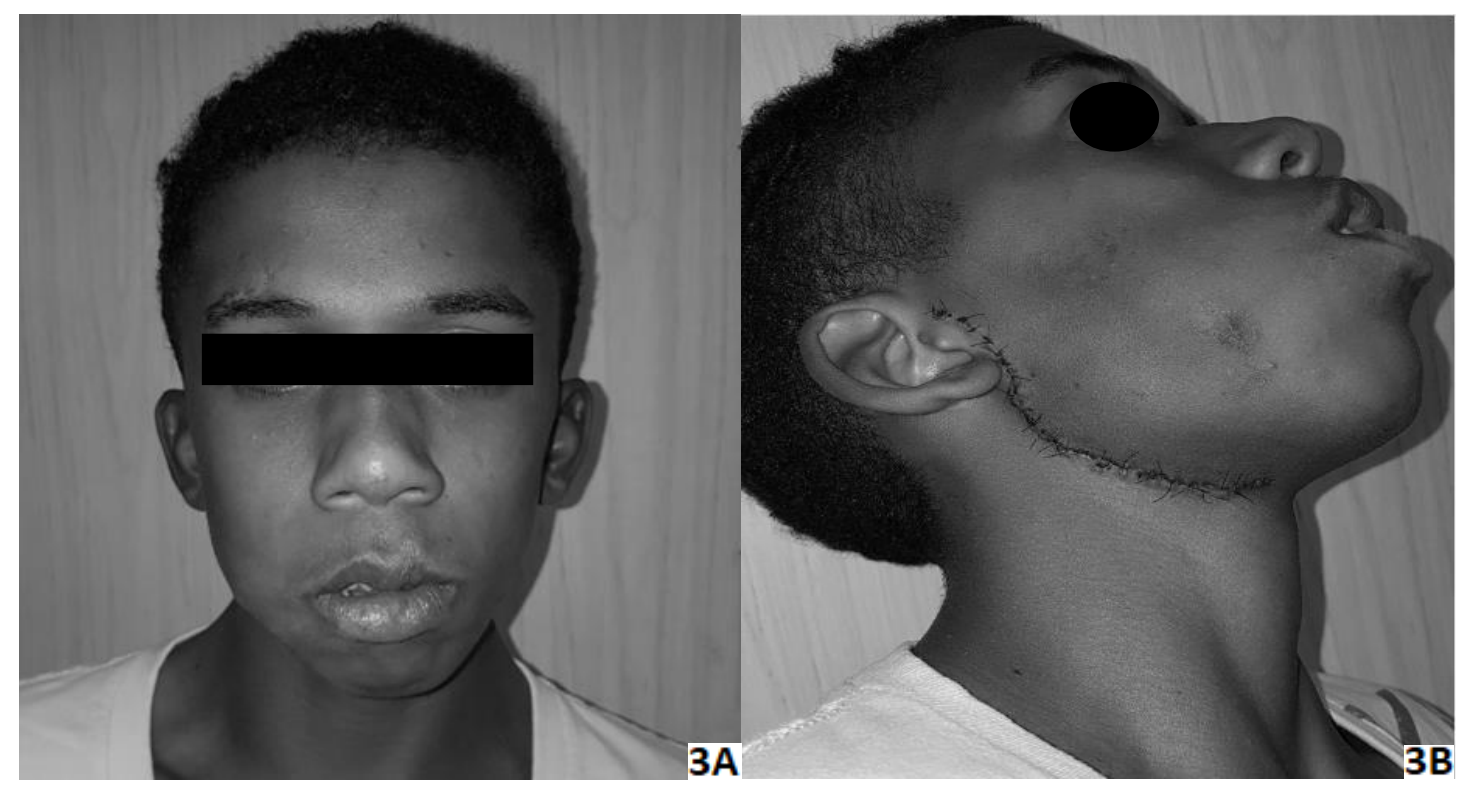

Figura 3. A) Dez dias pós-operatório, vista frontal; B) Dez dias pós-operatório, vista lateral.

\section{Discussão}

A patogênese das MAVs não é compreendida. A MAV intracraniana é a mais comum, seguida pelos locais extracranianos de cabeça e pescoço, extremidades, tronco e vísceras (MARLER, J. J., 2005). MAVs são erros raros de morfogênese vascular que estão presentes no nascimento, mas podem não ser clinicamente evidentes ou não apresentar um hemangioma definido como ciclo de vida. A lesão é composta por uma 
COPYRIGHT @ 2019 INTERNATIONAL JOURNAL OF SCIENCE DENTISTRY | AVAILABLE ONLINE http://www.periodicos.uff.br/index

massa de artérias e veias interconectadas confluentes sem plexo interposto e, portanto, apresenta um fluxo sanguíneo elevado (OKA, H., 2007).

O diagnóstico clínico é confirmado por ultrassonografia e exame Doppler colorido. A angiografia por ressonância magnética melhor documenta a extensão da malformação vascular, mostrando graus variáveis de dilatação e tortuosidade arterial, desvio arteriovenoso e veias de drenagem dilatadas. As artérias em alimentação podem apresentar aneurismas em pacientes idosos. Em alguns casos, fístulas discretas podem ser visualizadas na MAV difusa (MARLER, J. J., 2005).

Muitas abordagens cirúrgicas e não-cirúrgicas têm sido utilizadas para o tratamento de MAVs da cabeça e pescoço. O tratamento pode incluir técnicas endovasculares ou percutâneas para diminuir o fluxo sanguíneo e a probabilidade de hemorragia com vários agentes esclerosantes, incluindo partículas de álcool polivinílico, músculo, Gelfoam, cianoacrilato, e colágeno (PERROTT, D.H., 1994).

Ao usar técnicas de embolização, vários tratamentos podem ser necessários. A ligação da artéria carótida externa tem sido descrita como um complemento, mas a maioria dos autores desaconselha tal abordagem, porque muitas anastomoses promovem rápido aparecimento de circulação colateral (REMONDA, L., 1995). Além disso, essa abordagem impediria o tratamento futuro com angiografia e embolização (OKA, H., 2007).

No presente relato de caso, foi realizada a ressecção total da lesão durante o procedimento cirúrgico, visto que na angiografia realizada previamente à cirurgia não foi encontrado componente arterial significativo. Portanto, o resultado prévio do exame de imagem mostra-se essencial para guiar o tipo de tratamento que será realizado. No caso, não foram necessárias técnicas de embolização e foi utilizada unicamente a ressecção cirúrgica.

\section{Conclusão}

A ressecção cirúrgica da MAV foi considerada eficaz por não apresentar recidiva da lesão e resultados estéticos satisfatórios, entretanto, o paciente deverá ser acompanhado a fim de identificar quaisquer alterações de volume na área operada, visto 
COPYRIGHT @ 2019 INTERNATIONAL JOURNAL OF SCIENCE DENTISTRY | AVAILABLE ONLINE

http://www.periodicos.uff.br/index

a precocidade do acompanhamento. Podemos concluir também, que a inter-relação entre os serviços de Cirurgia Maxilofacial e Vascular foi essencial para um procedimento cirúrgico mais seguro e mais previsível.

\section{Referências Bibliográficas}

1. Lee BB, Baumgartner I, Berlien HP, Bianchini G, Burrows P, Do YS, et al. Consensus Document of the International Union of Angiology (IUA)2013. Current concept on the management of arterio-venous management. Int Angiol 2013;32:9-36.

2. Kohout MP, Hansen M, Pribaz JJ, Milliken JB. Arteriovenous malformations of the head and neck: natural history and management. Plast Reconstr Surg 1998 ; 102 :643-54 (PMID: 9727427).

3. Kumar A., Mittal M., Srivastava D., Jaetli V., Chaudhary S. Arteriovenous malformation of face. Contemp Clin Dent. 2017;8(July-September (3)):482484

4. Su L.X., Fan X.D., Zheng J.W., Wang Y.A., Qin Z.P., Wang X.K. A practical guide for diagnosis and treatment of arteriovenous malformations in the oral and maxillofacial region. Chin J Dent Res. 2014;17:85-89.

5. Marler, J. J., \& Mulliken, J. B. (2005). Current management of hemangiomas and vascular malformations. Clinics in Plastic Surgery, 32(1), 99-116. doi:10.1016/j.cps.2004.10.001

6. Oka, H., Pogrel, M. A., Dowd, C. F., \& Lee, J. S. (2010). Treatment of Arteriovenous Malformation of the Mandible With Resection and Immediate Reconstruction. Journal of Oral and Maxillofacial Surgery, 68(3), 658-663. doi:10.1016/j.joms.2007.12.033

7. Perrott DH, Schmidt B, Dowd CF, et al: Treatment of a highflow arteriovenous malformation by direct puncture and coil embolization. J Oral Maxillofac Surg 52:1083, 1994

8. Remonda L, Schroth G, Ozdoba C, et al: Facial intraosseous arteriovenous malformations: CT and MR features. J Comput Assist Tomogr 19:277, 1995 